\title{
Study of changes in microbiomes of sponges Lubomirskia baicalensis using the cell culture of primmorphs
}

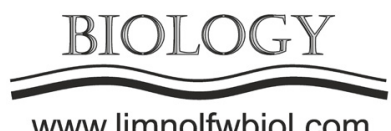

\author{
Chernogor L.I. ${ }^{1}$, Petrushin I.S. ${ }^{1,2}$, Khanaev I.V. ${ }^{1}$, Novolodskaya N.V. ${ }^{2}$, \\ Makarova Y.A. ${ }^{2}$, Belikov S.I. ${ }^{1}$
}

\author{
${ }^{1}$ Limnological institute, Siberian Branch of the Russian Academy of Sciences, Ulan-Batorskaya Str., 3 Irkutsk, 664033, Russia \\ ${ }^{2}$ Irkutsk State University, Karl Marx St, 1 Irkutsk, 664003, Russia
}

\begin{abstract}
Baikal freshwater sponges live in symbiosis with various species of eukaryotes and prokaryotes, including diverse chlorophyll-containing microalgae. Within the last years, there has been an increase in the detection of diseased and dead Baikal sponges. The etiology and ecology of these events remain unknown. The main aim of this work was to test the use of primmorph (L. baicalensis) cell cultures as a tool for investigating the microbiomes of sponges. We found that the microbiome community of healthy sponge and primmorphs formed a group separate from the community of diseased sponges and infected primmorphs. This confirms the suitability of the primmorphs cell culture as a model sponge system. Mass mortality of green symbionts (Chlorophyta) and a shift in the microbial communities of sponges/primmorphs have been discovered. By using primmorphs cell culture, it was possible to identify potential opportunistic bacteria that can work together, potentially enhancing their pathogenic action. The primmorphs system described here is a powerful model system for studying basic mechanisms of sponge disease development.
\end{abstract}

Keywords: Lubomirskia baicalensis, cell culture of primmorphs, symbionts, pathogen

Endemic freshwater sponges (Demosponges, Lubomirskiidae) dominate in Lake Baikal in the littoral zone at depths of 3 to $35 \mathrm{~m}$. They cover close to $50 \%$ of the available surfaces (Pile et al., 1997) and represent a complex consortium of many species of eukaryotes and prokaryotes (Sand-Jensen and Pedersen, 1994; Bil et al., 1999). The sponges are chlorophyll-containing freshwater organisms due to their association with various chlorophyll-producing algae (Latyshev et al., 1992; Bil et al., 1999). Within the last years, there has been an increase in the detection of diseased and dead Baikal sponges. The etiology and ecology of these events remain unknown. The main aim of this work was to test the use of primmorph (L. baicalensis) cell cultures as a tool for investigating the microbiomes of sponges. The development of a model to investigate the transmission of pathogenic agents from diseased sponges requires a detailed study of pathogen-host interactions in the environment. However, these experiments with sponges are difficult to perform under the natural conditions of Lake Baikal. We used the cell culture of primmorphs for experimental infection in vitro to identify changes in the microbiomes of diseased sponges. Another aspect of our work was to show that it is possible to use primmorphs in vitro to study microbiome communities in diseased sponges, with subsequent sequencing of $16 \mathrm{~S}$ rRNA gene fragments (Ghyselinck et al., 2013). Bioinformatics and statistical analyses were performed using the QIIME2 2019.1 pipelines (Bolyen et al., 2018).

We found that the microbiome community of healthy sponge and primmorphs formed a group separate from the community of diseased sponges and infected primmorphs. This confirms the suitability of the primmorphs cell culture as a model sponge system. Mass mortality of green symbionts (Chlorophyta) and a shift in the microbial communities of sponges/primmorphs have been discovered. Microbes in diseased sponges and infected primmorphs belonged mainly to the Bacteroidetes and Proteobacteria and were much more diverse at the family level. Among these, the families Flavobacteriaceae, Burkholderiaceae, and Moraxellaceae were dominant. We observed the increase in relative abundance of Flavobacteriaceae in the diseased sponges, especially in cultures of the infected primmorphs. The microbial community of a healthy group of sponge/ primmorphs was mainly composed of the phylum Cyanobacteria (Chlorophyta symbiont of Lubomirskia sp.) with a minimum abundance of bacteria, which indicates their healthy state. These unicellular eukaryotes entering the complex symbiotic communities of Baikal sponges produce a significant amount of carbohydrates, chlorophyll, fatty acids, and secondary 
metabolites (Latyshev et al., 1992; Bil et al., 1999). The symbiosis between algae and freshwater sponges is known to provide mutual benefits of photosynthesis, as oxygen and nutrients pass from algae to sponge and carbon dioxide and phosphate - from sponge to algae (Wilkinson and Garrone, 1980; Pita et al., 2018). The results of this research establish primmorphs as an in vitro model for sponge-microbe interactions, which will help to expand our understandings of the symbiotic relationships of microorganisms with freshwater Baikal sponges during mass mortality.

\section{Acknowledgements}

The following grant information was disclosed by the authors: Federal Agency of Scientific Organizations: 0345-2019-0002. Russian Foundation for Basic research: 18-04-00224.

The authors appreciate the help of the service staff of the National Scientific Center of Marine Biology FEB RAS (the former A.V. Zhirmunsky Institute of Marine Biology).

\section{References}

Bil K., Titlyanov E., Berner T. et al. 1999. Some aspects of the physiology and biochemistry of Lubomirskia baicalensis, a sponge from Lake Baikal containing symbiotic algae. Symbiosis 26: 179-191.

Bolyen E., Rideout J.R., Dillon M.R. et al. 2018. QIIME 2: Reproducible, interactive, scalable, and extensible microbiome data science. PeerJ Preprints 6. DOI: 10.7287/ peerj.preprints. $27295 \mathrm{v} 2$

Ghyselinck J., Pfeiffer S., Heylen K. et al. 2013. The effect of primer choice and short read sequences on the outcome of 16S rRNA gene based diversity studies. PLoS ONE 8. DOI: 10.1371/journal.pone.0071360

Latyshev N.A., Zhukova N.V., Efremova S.M. et al. 1992. Effect of habitat on participation of symbionts in formation of the fatty acid pool of freshwater sponges of Lake Baikal. Comparative Biochemistry \& Physiology 102B: 961-965. DOI: 10.1016/0305-0491(92)90109-5

Pile A.J., Patterson M.R., Savarese M. et al. 1997. Trophic effects of sponge feeding within Lake Baikal's littoral zone. 2. Sponge abundance, diet, feeding efficiency, and carbon flux. Limnology and Oceanography 42: 178-184. DOI: 10.4319/ 10.1997.42.1.0178

Pita L., Rix L., Slaby B.M. et al. 2018. The sponge holobiont in a changing ocean: from microbes to ecosystems. Microbiome 6. DOI: 10.1186/s40168-018-0428-1

Sand-Jensen K., Pedersen M.F. 1994. Photosynthesis by symbiotic algae in the freshwater sponge, Spongilla lacustris. Limnology and Oceanography 39: 551-561. DOI: 10.4319/ lo.1994.39.3.0551

Wilkinson C., Garrone R. 1980. Nutrition of marine sponges. Involvement of symbiotic bacteria in the uptake of dissolved carbon. In: Smith D.C., Tiffon Y. (Eds.), Nutrition in the lower Metazoa. Oxford, pp. 157-161. 